\title{
Do Strollers in Town Needs Recommendation?: On Preferences of Recommender in Location-Based Services
}

\author{
Kenro Aihara \\ National Institute of Informatics \\ 2-1-2 Hitotsubashi, Chiyoda-ku, Tokyo 101-8430, Japan \\ kenro.aihara@nii.ac.jp
}

\begin{abstract}
When we discuss about recommendation especially in Location-Based Services (LBS), we need to reveal whether users really want recommendations or not in fact while they are strolling in town, prior to evaluate each recommendation model.

In this paper, a Location-Based Service, called nicotoco, is shown. nicotoco is an iPhone-based LBS in Futako-tamagawa area, Tokyo, Japan and provides information about stores and events to users. In the experiment using nicotoco, recommendations may be preferred more than rankings which was made from access counts.
\end{abstract}

Keywords: context-aware computing, location-based service, behavioral cost.

\section{Introduction}

A lot of recommender systems have been proposed for strollers [8]. Almost all of them seem to be location-aware and assume that the nearer the provided information is located, the more useful it is for users. This assumption implies that the information that may be preferable for the user but located a little further away vanishes from the user because of a massive amount of information, such as micro blogs like tweets of Twitter. On the other hand, although a recommender system can filter out the information that is probably uninteresting to the user based on the collaborative filtering model that is broadly used in existing recommender systems on the Internet, the author supposes that the information that may be preferable to the user must depend on the user's situation and the collaborative filtering model is still naive, especially when the user is strolling around in town.

In addition, when we discuss about recommendation especially in LocationBased Services (LBS), we need to reveal whether users really want recommendations or not in fact while they are strolling in town, prior to evaluate each recommendation model. 


\section{Background}

\subsection{Location-Based Information Services}

A lot of network services with location data are proposed, and some of them, such as foursquare 1 , are getting popular. Usually location information is given as geographical coordinates, that is, latitude and longitude, a location identifier such as ID for facilities in geographical information services (GIS), or a postal address. Google has launched Google Places2, which gathers place information from active participating networkers and delivers such information through Google's web site and API (application programmable interface). Google may try to grasp facts and information on activities in the real world where it has not enough information yet even though it seems to have become the omniscient giant in the cyber world. Google already captures some real world phenomena in its own materials. For example, it gathers landscape images with its own fleet of specially adapted cars for the Google Street View service 3 . However, the cost of capturing and digitizing facts and activities in the real world is generally very expensive if you try to obtain more than capturing photo images with geographical information. Although Google Places may be one of the reasonable solutions to gathering information in the real world, it's not guaranteed that it can grow into an effective and reliable source reflecting the real world.

Existing social information services, such as Facebook and Twitter, are expanding to attach location data to users' content.

\section{$2.2 \quad$ Filtering Information for Strollers}

In the field of recommender systems, collaborative filtering is one of the popular methods to judge whether information fits the user or not 6152 . The collaborative filtering model is basically based on the assumption that similar users prefer the same information. However, when we consider recommending information to mobile users who are strolling in town, the author believes that the information must be selected further from a set of already filtered candidates in accordance with their situation because the input method and output devices of mobile terminals are highly restricted and also the number of candidates still has to be large even though they are already filtered.

\subsection{Phenomena of Human's Preference}

In the field of behavioral economics, the phenomena of time preference and temporal discounting are known, which refer to a decrease in the subjective value of a reward as the delay of its receipt increases [4]. People and other

\footnotetext{
1 http://foursquare.com/

2 http://www.google.com/places/

3 http://www.google.com/streetview/
} 


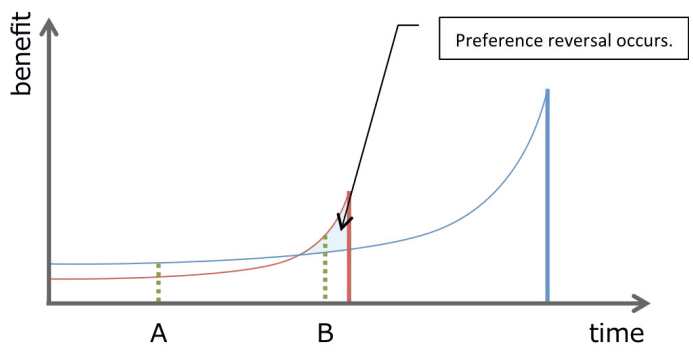

Fig. 1. Preference reversal phenomenon

animals discount future reward as a function of time. In addition, there is another remarkable phenomenon of preference reversal, which occurs when a subject places a lower selling price on the gamble that he/she chooses than on the other gamble in a pair [7. Animal and human temporal discounting has been described better as hyperbolic functions than exponential ones in recent psychology (Figure 11).

This notion implies that humans prefer not always rational choices but sometimes irrational and impulsive ones, especially in stressful situations. The author proposed a recommendation model based on this notion for strollers in town [1].

\section{Nicotoco: An LBS for Strollers}

\subsection{Service Description}

"niconoto" is supplied since the end of November, 2011. The service is designed for strollers who visit Futako-tamagawa area, which is being redeveloped as a smart city in Tokyo and consists of complexes including shopping malls, supermarkets, offices, and residential areas around the Futako-tamagawa station. The service can be accessed both via web browser and via iPhone application. When visitors arrive in the service area and access the service, they can get information according to their location and their own user model that is learned from users' attributes and behavior logs.

Major functions of the service are as follows.

Top Page. When users access the top page of the service with web browser, the page gives general information including hot events, up-to-date tweets from stores, and recommended spots, such as stores.

If the user uses the iPhone application, three recommended spots are given (Figure 2(a)). Users also see more recommendations to tap “もつとみる” (means "see more") button at the bottom right on the screen. And also users can switch the list to the rankings of mostly accessed spots in recent days (Figure 2(b) by tapping the medal icon on the top right. 


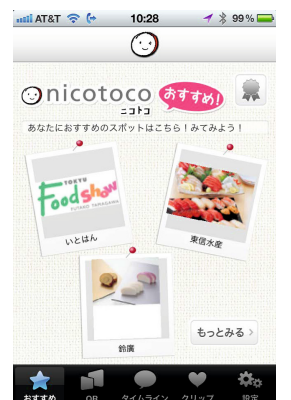

(a) Recommendation

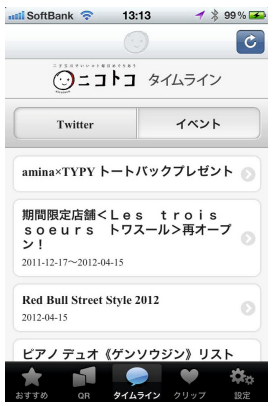

(e) Hot events

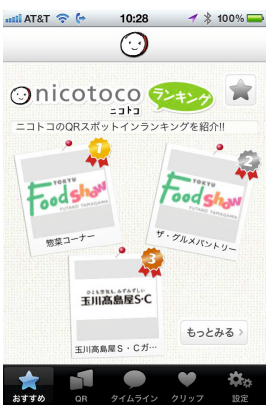

(b) Ranking

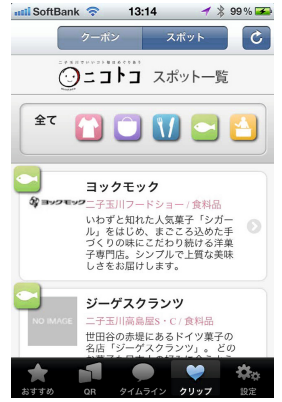

(f) Clipped spots

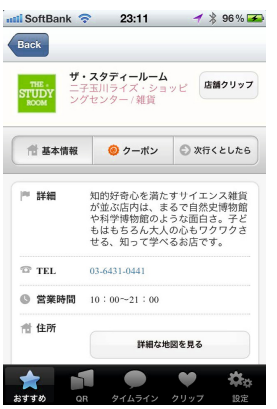

(c) Spot page

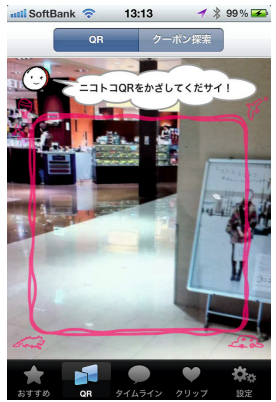

(g) QR scanner

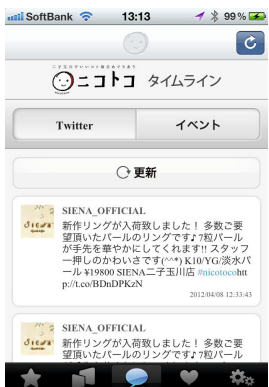

(d) Time line

Fig. 2. Snapshot images of the nicotoco application

Spot Information. When a user selects one spot on the screen, the system shows an information including maps, coupons if available, tweets from the store (Figure 2(c), Users can get further recommendation of spots the very next candidates to the spot by tapping “次いくとしたら” ("Where to Go Next?").

Time Line. The application gives two kinds of time lines: tweets (Figure 2(d)) and events (Figure 2(e) .

Clips. Users can clip spots and coupons when they see the correspondings page just like as bookmarking. Clipped spots and coupons can be reviewed in "クリップ" ("Clip") mode in the bottom menu (Figure 2(f)]. In a typical scenario, users once check favorite spots in recommendations or rankings and then clip them. When they visit the spot and show the clipped coupon to get discount or corresponding service.

Spot Check-in with QR Code Markers. In addition to the basic functions above, the service deploys sticker markers at affiliates through the area. The 


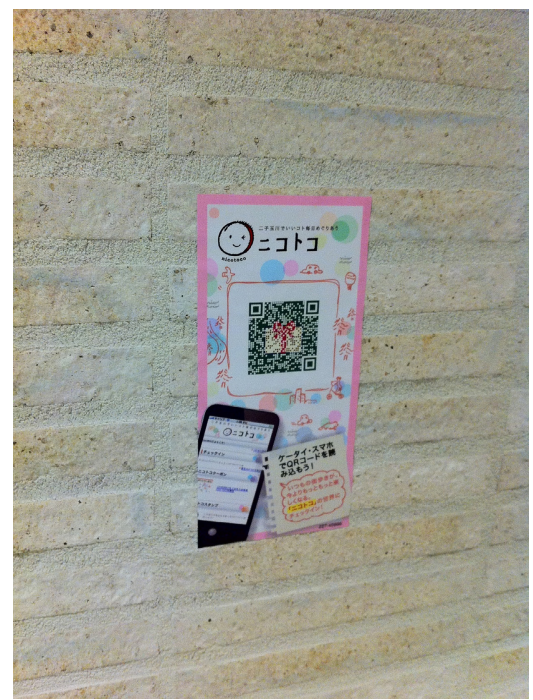

(a)

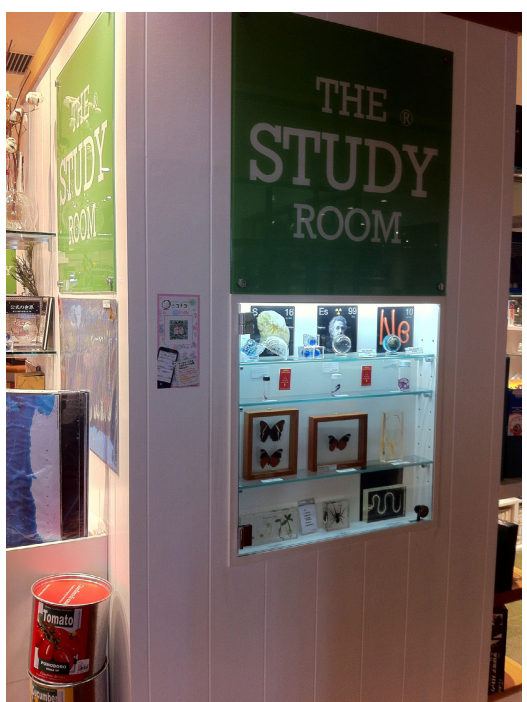

(b)

Fig. 3. QR Code Markers for Spot Check-in

marker shows the serivce logo and QR code, which is a popular two-dimensional code and it can be scanned and decoded by almost all of cell phones sold in Japan. When a user scans a code in nicotoco marker with one of standard QR readers, the information corresponding to the marker will be shown. If the store delivers coupons for the service customers, the user can get the coupon and the coupon is stored for recall when he/she uses. Figure 2(g) shows an image of scanner mode for QR in the application. Examples of QR code markers are shown in Figure 3 .

The action of scanning the code is also regarded as "check-in" for the spot.

AR View. In the iPhone application, AR mode is equipped. In AR mode, icons of the spots that deliver coupons overlays onto the captured image of the iPhone camera in real time to search stores around the user (Figure 2(h)).

\subsection{User Data}

The nicotoco service collects user data as follows:

- attributes

- gender

- generation

- zip code

- behavior logs 
- access logs for selected spots

- check-ins for spots (readings of markers)

- locations (application only)

The nicotoco service collects users' demographic attributes at the first access, behavior logs including access logs to the service and their locations sensed with GPS and Wi-Fi signals when it is active and their location gets updated.

\subsection{Process of Recommendation}

Feature Value of Users. Users are characterized by vector of attributes and terms which occur in their own contents, such as tweets, and features of spots where they visited. That is, feature of each user will be updated by visiting spots.

Feature of user $u$ at the current time $c_{c u r}$ is defined in Equation 1 . readmarker $(u, s, t)$ denotes the log count of reading markers of spot $s$ by user $u$ at the time $t$.

$$
\begin{gathered}
\overrightarrow{u s e r}\left(u, t_{\text {cur }}\right)=(\text { gender, generation }, \overrightarrow{\text { visit }}) \\
\overrightarrow{v i s i t}=\sum_{s}^{S} \sum_{t \in t s}^{t_{c u r}}\left(\text { decay }(t) \cdot \operatorname{readmarker}(u, s, t) \cdot \overrightarrow{\operatorname{spot}}\left(s, t_{\text {cur }}\right)\right) \\
t s=\operatorname{timeslot}(t) \\
\operatorname{decay}(t)=\exp \left(t-t_{\text {cur }} / C_{t}\right)
\end{gathered}
$$

$C_{t}$ denotes a constant of period for decay function. 12 time slots $t s$ are provided: $12 \mathrm{am}-4 \mathrm{am}, 4 \mathrm{am}-10 \mathrm{am}, 10 \mathrm{am}-12 \mathrm{pm}, 12 \mathrm{pm}-3 \mathrm{pm}, 3 \mathrm{pm}-6 \mathrm{pm}, 6 \mathrm{pm}-9 \mathrm{pm}$, and 9pm$12 \mathrm{am}$ of weekdays and weekends, respectively.

Feature Value of Spots. Spots are characterized by vector of terms which occur in their own descriptions and features of users who visited them, as well. That is, feature of each spot will be updated by users' visits. $\overrightarrow{d e s c}$ denotes feature value based on term frequency in descriptions on the spot.

$$
\begin{gathered}
\overrightarrow{s p o t}\left(s, t_{\text {cur }}\right)=(\overrightarrow{\text { desc }}, \overrightarrow{\text { visited }}) \\
\overrightarrow{\text { visited }}=\sum_{u}^{U} \sum_{t \in t s}^{t_{\text {cur }}}\left(\operatorname{decay}(t) \cdot \text { readmarker }(u, s, t) \cdot \overrightarrow{u s e r}\left(u, t_{\text {cur }}\right)\right)
\end{gathered}
$$

User's Commitment to Spots. The commitment of user $u$ to spot $s$ at time slot of the current time $t_{\text {cur }}$ is given from behavior logs by Equation $3, \alpha, \beta, \gamma$ are coefficients of linear combination.

$$
\operatorname{cmt}(u, s, t s)=\alpha \cdot v i s(u, s, t s)+\beta \cdot \operatorname{acc}(u, s, t s)+\gamma \cdot c p n(u, s, t s)
$$




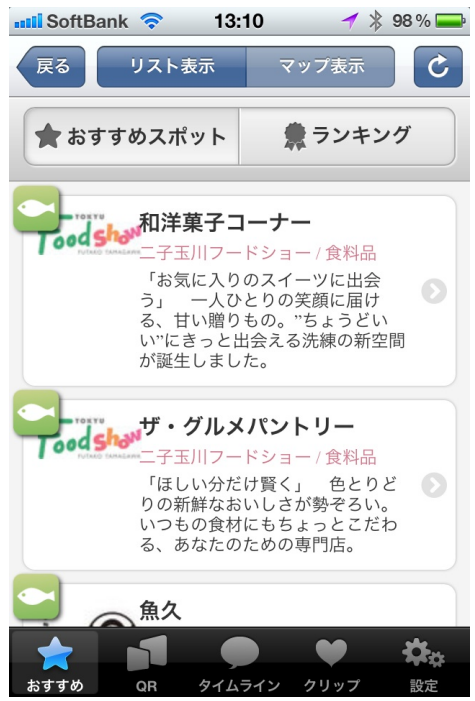

Fig. 4. List of Recommendation of the nicotoco application

$$
\begin{aligned}
\operatorname{vis}(u, s, t s) & =\sum_{t \in t s}^{t_{\text {cur }}}(\operatorname{decay}(t) \cdot \text { readmarker }(u, s, t)) \\
\operatorname{acc}(u, s, t s) & =\sum_{t \in t s}^{t_{\text {cur }}}(\operatorname{decay}(t) \cdot \operatorname{accesspage}(u, s, t)) \\
\operatorname{cpn}(u, s, t s) & =\sum_{t \in t s}^{t_{\text {cur }}}(\operatorname{decay}(t) \cdot \operatorname{accesscoupon}(u, s, t))
\end{aligned}
$$

Transitivity between Spots. Transitivity $\operatorname{tr}\left(u, s_{i}, s_{j}, t s\right)$ from spot $s_{i}$ to spot $s_{j}$ of user $u$ in time slot $t s$ is calculated from occurrences of spot transitions within given interval in users' behavior logs.

Recommendation of Spots. By using $\overrightarrow{u s e r}\left(u, t_{c u r}\right), \overrightarrow{s p o t}\left(s, t_{c u r}\right)$, and $c m t(u, s, t s)$, relativity between user $u$ and spot $s$ in time slot $t s$ are calculated in Equation 4 .

$$
\begin{aligned}
\operatorname{score}(u, s, t s)= & C \cdot \cos \left(\overrightarrow{u s e r}\left(u, t_{\text {cur }}\right), \overrightarrow{\operatorname{spot}}\left(s, t_{\text {cur }}\right)\right) \\
& +(1-C) \cdot \operatorname{cmt}(u, s, t s)
\end{aligned}
$$

Top $k$ spots with higher relativity scores are given as recommendations as shown at top page (Figure 2(a). In addition, more recommendation can be called (Figure 4). 
Table 1. Statistics of the nicotoco service of iPhone application

\begin{tabular}{|c|c|c|c|}
\hline \multicolumn{2}{|c|}{ type of action } & \multicolumn{2}{|c|}{ |number of unique users|number of actions } \\
\hline \multicolumn{2}{|c|}{ activate the application } & 243 & 9935 \\
\hline \multicolumn{2}{|c|}{ get recommendation listing } & 324 & 1997 \\
\hline \multicolumn{2}{|c|}{ picked spots } & 138 & 294 \\
\hline & "go next" & 93 & 140 \\
\hline \multirow[t]{2}{*}{ get ranking } & listing & 111 & 623 \\
\hline & picked spots & 32 & 113 \\
\hline \multirow[t]{4}{*}{ use Camera mode } & activate $\mathrm{QR}$ scanner & 46 & 130 \\
\hline & scanned QR & 19 & 131 \\
\hline & $\mathrm{AR}$ view & 80 & 209 \\
\hline & picked spots in AR & 22 & 193 \\
\hline
\end{tabular}

Recommendation of Where to Go Next. In addition to recommendations mentioned above, the nicotoco service gives another type of recommendation which aims to give suggestions where to go next when users visit a spot. In many usual recommenders, users can get suggestions relative to their favorites; that is, similar contents can be provided. For example, a user who likes cafe tends to get recommendations from cafes. The author, however, supposes that a user who just visited a cafe doesn't want recommendation of another cafe and may prefer somewhere favorite in another category. In nicotoco, such recommendations of where to go next are calculated by using transitivity between spots in addition to scores between user and spot (Equation [5). $t r$ denotes transitivity from spot $s_{i}$ to spot $s_{j}$. score denotes recommendation score mentioned above.

$$
\begin{aligned}
& n s c o r e\left(u, s_{\text {org }}, s, t s\right)=C_{n} \cdot \operatorname{tr}\left(u, s_{\text {org }}, s, t s\right) \\
& +\left(1-C_{n}\right) \cdot \operatorname{score}(u, s, t s)
\end{aligned}
$$

Top $k$ recommended spots are shown when users tap “次いくとしたら” button as shown in Figure 2(c).

\section{Statistics of the Service}

Web-based service was launched at the end of November, 2011. 3,239 users are registered and 2,399 users accessed the service, as of the end of February, 2012. Male users are 803; female, 1,594; missing, 2.

For the iPhone application, it got ready to be downloaded from AppStore of Apple Inc. in February, 2012. The number of users is 357 (male is 192; female, 165). At first, 138 of 324 users (42.6\%) picked recommended spots at least once. And also the number of unique users who used "Go Next" function is 93 (36.3\%). The author believes that these results are able to support that recommendation function is potentially prefered in LBSs. In particular, the preference of "Go Next" relatively may be higher, considering the accessibility of the mode against normal recommendation listing. 
In comparison with ranking, recommendation seems much preferred. This insight may be interesting because ranking is usually based on general popularity and users prefer personalized or "tailored" information more than such popularity. It may implies that users cannot be satisfied with "Like" count-based ranking.

\section{Conclusions}

The paper shows the service called nicotoco, which facilitates the model for recommendation. In results of statistics of the nicotoco service, the author believes that recommendation function can be preferred by strollers using LBSs.

The author continues to develop and provide these services. To evaluate the effectiveness of the model, experiments are being planned. Analysis of user behavior logs and the development of methods to capture users' situation including cognitive aspects are future issues.

Acknowledgments. The author thanks Tokyu Corporation and Kokusai Kogyo Co., Ltd. for their cooperation with this research. This work is partly supported by the Ministry of Economy, Trade and Industry of Japan.

\section{References}

1. Aihara, K., Koshiba, H., Takeda, H.: Behavioral cost-based recommendation model for wanderers in town. In: Jacko, J.A. (ed.) Human-Computer Interaction, Part III, HCII 2011. LNCS, vol. 6763, pp. 271-279. Springer, Heidelberg (2011)

2. Braak, P.T., Abdullah, N., Xu, Y.: Improving the performance of collaborative filtering recommender systems through user profile clustering. In: Proceedings of the 2009 IEEE/WIC/ACM International Joint Conference on Web Intelligence and Intelligent Agent Technology, vol. 3, pp. 147-150 (2009)

3. Ducheneaut, N., Partridge, K., Huang, Q., Price, B., Roberts, M., Chi, E.H., Bellotti, V., Begole, B.: Collaborative filtering is not enough? experiments with a mixed-model recommender for leisure activities. In: Houben, G.-J., McCalla, G., Pianesi, F., Zancanaro, M. (eds.) UMAP 2009. LNCS, vol. 5535, pp. 295-306. Springer, Heidelberg (2009)

4. Green, L., Fry, A.F., Myerson, J.: Discounting of delayed rewards: A life-span comparison. Psychological Science 5(1), 33-36 (1994)

5. Schafer, J.B., Frankowski, D., Herlocker, J., Sen, S.: Collaborative filtering recommender systems. In: Brusilovsky, P., Kobsa, A., Nejdl, W. (eds.) Adaptive Web 2007. LNCS, vol. 4321, pp. 291-324. Springer, Heidelberg (2007)

6. Su, X., Khoshgoftaar, T.M.: A survey of collaborative filtering techniques. Advances in Artificial Intelligence 2009, 1-19 (2009)

7. Tversky, A., Kahneman, D.: the framing of decisions and the psychology of choice. Science 211(4481), 453-458 (1981)

8. Zheng, V.W., Cao, B., Zheng, Y., Xie, X., Yang, Q.: Collaborative filtering meets mobile recommendation: A user-centered approach. In: Proceedings of the 24th AAAI Conference on Artificial Intelligence, pp. 236-241 (2010) 